\title{
Tree root detection from ground surface vibration measurements
}

\author{
Michał K. Kalkowski ${ }^{1,2, \star}$, Jennifer M Muggleton¹, and Emiliano Rustighi ${ }^{1}$ \\ ${ }^{1}$ Institute of Sound and Vibration Research, Faculty of Engineering and the Environment, University of Southampton SO17 1BJ, \\ UK \\ ${ }^{2}$ Department of Mechanical Engineering, Imperial College, London SW7 2AZ, UK
}

\begin{abstract}
Rapid development of urban infrastructure in past decades together with a relatively recent growth of awareness of its impact on the natural environment result in an increased interest in non-destructive ground interrogation methods. Tree root damage is a very well known issue in civil engineering and can emerge as road surface fracture, building foundations disintegration or pipe penetration, among others. In this paper we investigate the feasibility of using a vibroacoustic method for tree root mapping. The core of the idea is that the mechanical waves induced by an excitation mechanism acting on the tree trunk propagate to the roots and then radiate into the surrounding soil. Owing to that, the response measured at the ground surface contains the contribution of waves radiating from roots and can be used for mapping their extent. In this paper, we report a set of field experiments on a 'purpose-built' root-trunk model buried underground. These preliminary results both demonstrate the technique and shed light on related challenges and limitations.
\end{abstract}

\section{Introduction}

Trees play a vital role in the carbon cycle, through oxygen synthesis and biomass production. However, in the era of rapid urbanisation and climate changes, tree ecosystems are endangered. On one hand it is very much desired that cities contain large green areas, but at the same time, intense exploitation of city space is a threat for trees. Particularly challenging aspect is the coexistence of tree root networks with city infrastructure. Roots' growth often results in an unexpected damage to pipe networks, road surfaces and building foundations. On the other hand, roots can be severely damaged by building works. It is essential that the location and growth direction of living roots can be monitored periodically, to allow for an informed construction planning and infrastructure maintenance.

There have been a number of attempts to develop remote root mapping techniques, but probably the only approaches that received considerable attention are based on the geotechnical penetrating radar (GPR) [1] and resistivity imaging [2]. Despite promising results reported, several limitations remain, including a complicated measurement setup (resistivity), sensitivity to weather/moisture conditions, common artefacts, or data processing requiring highly expert algorithms and/or operator [3-6].

In this paper, we investigate the possibility of using vibration to detect tree roots from ground surface vibration measurements. Conceptually, this research originates from vibroacoustic water pipe mapping which our research group has pioneered for past decades $[7,8]$. The approach is based on sensing waves re-radiated by externally excited underground structures. In the considered

\footnotetext{
^e-mail: m.kalkowski@imperial.ac.uk
}

case, the excitation is applied to the tree trunk. Wave energy travels down to the root network, is re-radiated into the surrounding ground and eventually propagates to the ground surface, where it can be sensed. This technique has proved itself successful for locating water pipes, as reported in the aforementioned references.

In this contribution, we present an experimental setup involving a mock up trunk-root system, which is used to investigate how root-like structures radiate waves to the ground surface. This is followed by some preliminary measured results from two types of sensor arrays; it is noted that we analyse 'bare' dynamic responses in this work and do not employ any advanced signal processing. Finally, we draw a number of observations directing current ant future work on this topic.

\section{Field test rig set-up}

Given the complexity of the problem, it was concluded that a fundamental physical insight may be gained from a controlled experiment. We built a mock up trunk-root system, which was equipped with a number of sensors and buried in the ground. In this following paragraphs, the rig used for our investigation is described in more detail.

\subsection{Root}

The root was represented by its simplest physical model - an exponentially tapered wooden bar $(\approx 2 \mathrm{~m}$ long with radius varying exponentially from $0.0945 \mathrm{~m}$ to $0.0035 \mathrm{~m}$ ). Arborists indicate that the change of diameter of the main roots in the vicinity of the trunk can be characterised by an 


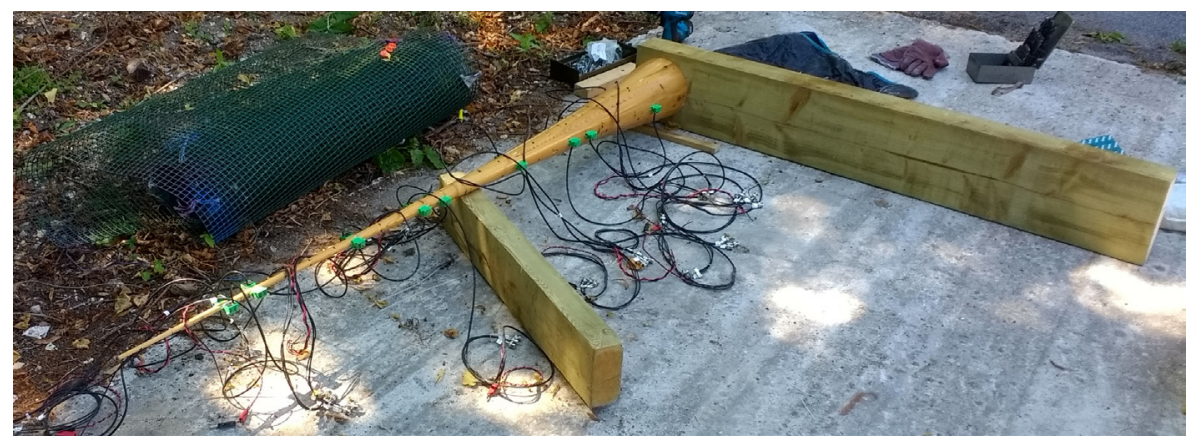

Figure 1. Trunk-root assembly before installation.

exponential profile and is called a 'zone of rapid taper' [9]. Compared to real roots, our setup involved two significant approximations; firstly, we neglected curvature typical for real roots and secondly, the specimen had a non-realistic material structure. Tree roots are cylindrically orthotropic; we used a wooden specimen, but owing to the complexity associated with sourcing such a large block of wood, our model was a glued assembly of several parts which did not preserve the cylindrical symmetry. Nevertheless, we believed that such a configuration is representative for the purpose of the investigation. Prior to the experiment, we measured wave characteristics of a free root in the laboratory and confronted them with a wave propagation model, obtaining very good agreement [10].

\subsection{Trunk}

The trunk was represented by a piece of timber $(0.1$ $\times 0.225 \times 1.5 \mathrm{~m}$ ) which was screwed and epoxy-bonded to the root after the former had been installed in the ground. The trunk hosted a shaker and an accelerometer placed next to the shaker for the purpose of recording input acceleration. The complete root-trunk assembly before installation is shown in Fig. 1.

\subsection{On-root sensors}

Our interest was in measuring both the ground surface response and the response at the root surface. For this purpose, we equipped the root with a number of in-house assembled MEMS accelerometers. The choice of the sensors had a two-fold motivation. Firstly, there was a need for perfectly waterproof sensors which can operate buried in the soil under different environmental conditions. Secondly, it seemed appealing to verify whether low-cost sensors can be used for scientific investigation. We used widely available GY-61 boards equipped with triaxial ADXL335 accelerometer chip. After changing respective capacitors to widen the bandwidth, the boards were placed in 3D-printed enclosures, facilitating installation on the root and potted using an epoxy-based compound. The sensors were installed at two opposite sides of the root and at nine locations along its axis, giving 18 triaxial accelerometers buried underground altogether (visible in Fig. 1.

\subsection{Field installation}

The mock-up trunk-root system was installed at a research site at Chilworth, owned by the University of Southampton. The root was buried at the depth of $0.5 \mathrm{~m}$, with sand lining in the immediate vicinity of the root (to ensure better contact with the ground and protect the sensors) (Fig. 2). The trench was filled back with soil and compacted successively using a trench rammer. During installation it was discovered that the soil was very inhomogeneous at that site, with a large number of stones and hair-thin roots (Fig. 2 (a)). Such structure, although likely to occur in practical settings, surely is an additional challenge for our investigation. At first, a small access hole was left around the large end of the root. This was used for a series of measurements aimed at characterising waves in a buried exponential structure as such, which are not reported in this paper. After these measurements were taken, the trunk was attached using a number of heavy-duty screws and epoxy, as depicted in Fig. 2 (b).

\subsection{Ground surface sensors}

The response at the ground surface was recorded using SM-24 tri-axial geophones (see Fig. 2 (d)) arranged in two kinds of array (see Fig. 3). The first was a rectangular array with eight locations along the root axis and nine locations perpendicular to it. The second was a circular array, with seven geophones arranged radially, at approximately every 30 degrees around the trunk. The former configuration is typical for pipe measurements reported above, but the latter seems to correspond better to the current application context.

\section{Experimental procedure}

Our first investigation was aimed at verifying whether the signatures of the root are present in the ground surface response to trunk excitation. For this reason a number of frequency response functions were recorded using a PROSIG P8020 system. As the site was located in a business park, very close to a compressor station and other facilities, measured ground vibration responses were found to be very noisy. To address the low signal-to-noise issue, we used stepped sine excitation and extracted the magnitude and phase at every frequency step from Fourier series 
(a)

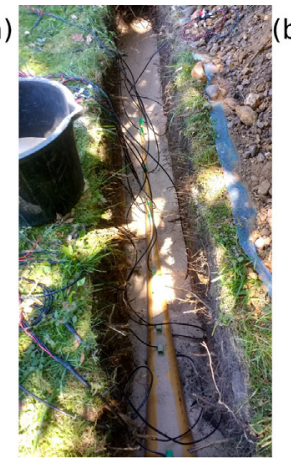

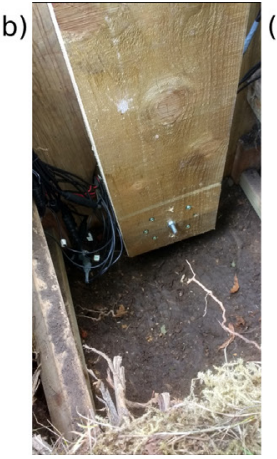
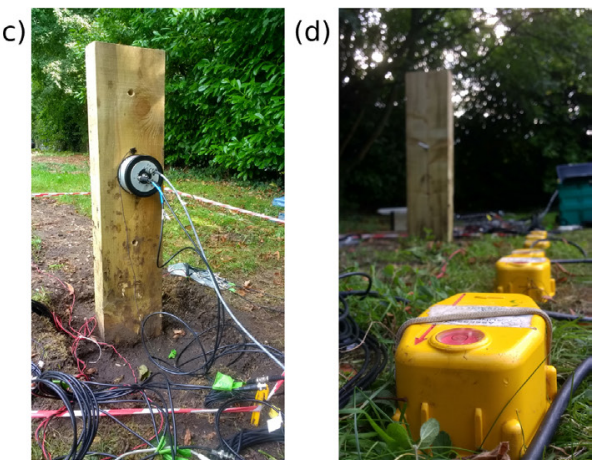

Figure 2. Field installation: (a) inhomogeneous structure of the soil and root installation; (b) trunk attachment; (c) shaker on the trunk; (c) ground surface sensors.
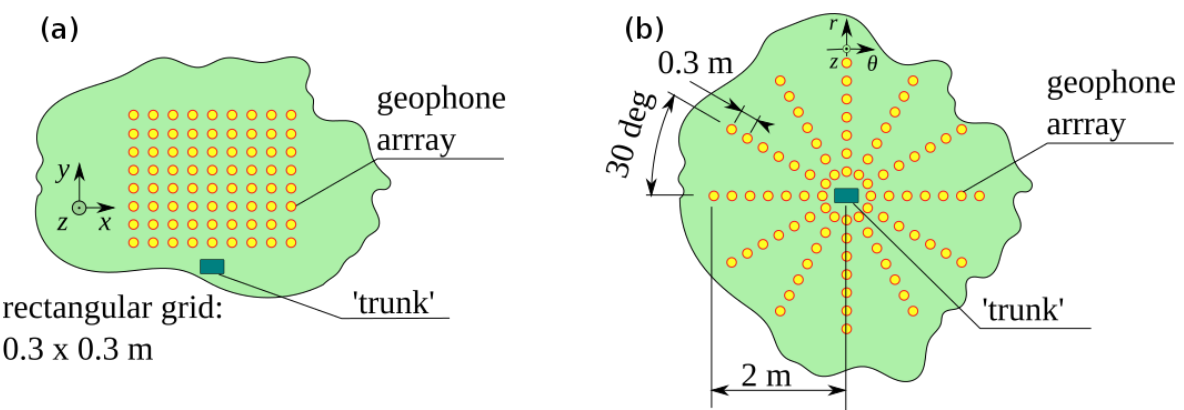

Figure 3. Two array configurations used in the experiment: (a) rectangular; (b) circular.

coefficients. Limited by a number of geophones available, the recording needed to be repeated a number of times to populate the array. However, given that measurements for a single array were taken very close in time with the same excitation setup and under approximately constant environmental conditions, we did not expect them to introduce any noticeable variability. For both geophone arrays, the trunk was excited along three orthogonal excitation directions using a Wilcoxon Research (WR) F4 shaker with a dedicated WR amplifier.

\section{Results}

Wave-based buried utility mapping uses unwrapped phase maps to identify the objects of interest, as reported in $[7,8]$. Adopting an appropriate phase unwrapping scheme is an important factor associated with the method. For our considered problems of tree roots, we used a number of spatial approaches, but none of them was found to bring clear results. Consequently, in this paper we only present some magnitude graphs, in which root signatures can be observed. While analysing ground vibration response maps, one needs to use some criterion for the choice of frequencies to look at. Our first, rather simplistic choice, was to focus on peaks of the frequency response functions averaged over the whole array.

\subsection{Rectangular array}

Two sets of illustrative results - for the axial $(y)$ and transverse $(x)$ excitation - recorded with the rectangular array are shown in Fig. 4. The signatures of the root are clearly visible in the total superimposed response $u_{\text {total }}$ (vector sum of all directional components), but for the axial excitation the signature is shifted with respect to its actual location. A possible factor contributing to this is that the soil just above the root was disturbed and mechanically compacted, which possibly introduced further macro-scale inhomogeneity into the soil. One remaining concern is that the response at the opposite side to the trunk is unknown. Given the type of excitation, it is expected that the response would visually resemble a dipole, which may misguide the interpretation. This is expected to be addressed with the circular array.

The chosen response to transverse excitation is slightly more convincing - the $x$-component of the response is clearly dominated by waves re-radiated from the root. Both graphs indicate the potential of vibration-based methods for tree root mapping.

\subsection{Circular array}

Using a circular array showed that, the blue spot around 40 $\mathrm{Hz}$ is, indeed, the signature of the root (see Fig. 5). Interestingly, at higher frequencies, it becomes more focused, clearly aligning with the line of the root. As expected, moving towards shorter wavelenghts increases the resolution of detection ( $u_{r}$ response in Fig. 5).

Illustrative responses for the two other directions are shown in Fig. 6. For transverse excitation the root is visible at $57 \mathrm{~Hz}\left(u_{\theta}, u_{\text {total }}\right)$, but it appears at an incorrect angle. 
(a)

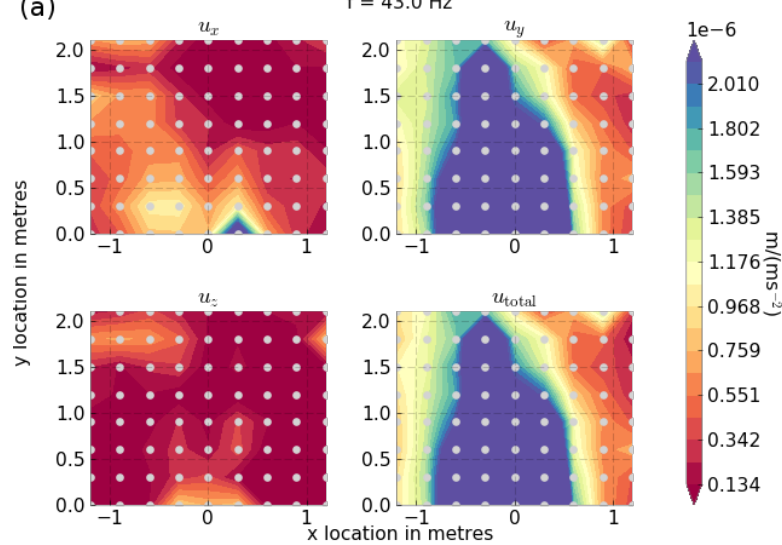

(b)

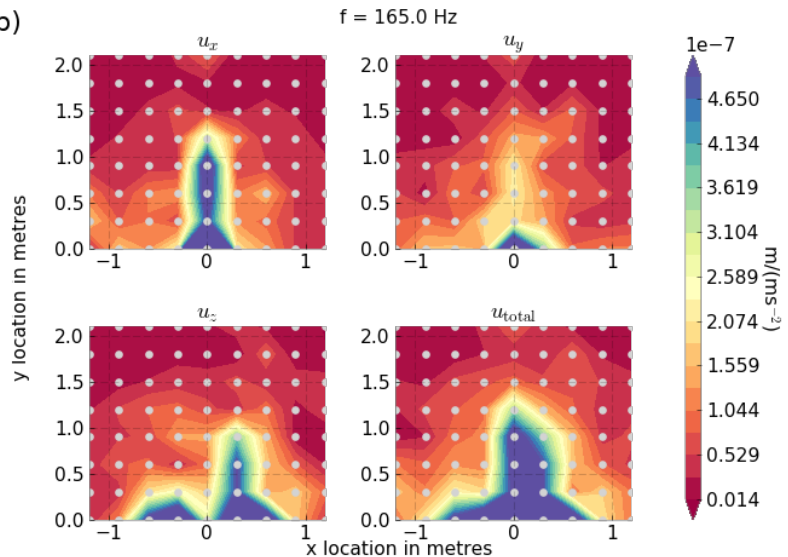

Figure 4. Illustrative magnitude maps for the rectangular array; the root is located along the $y$-direction, at $x=0$ : (a) axial excitation at $43 \mathrm{~Hz}$; (b) transverse excitation at $165 \mathrm{~Hz}$.
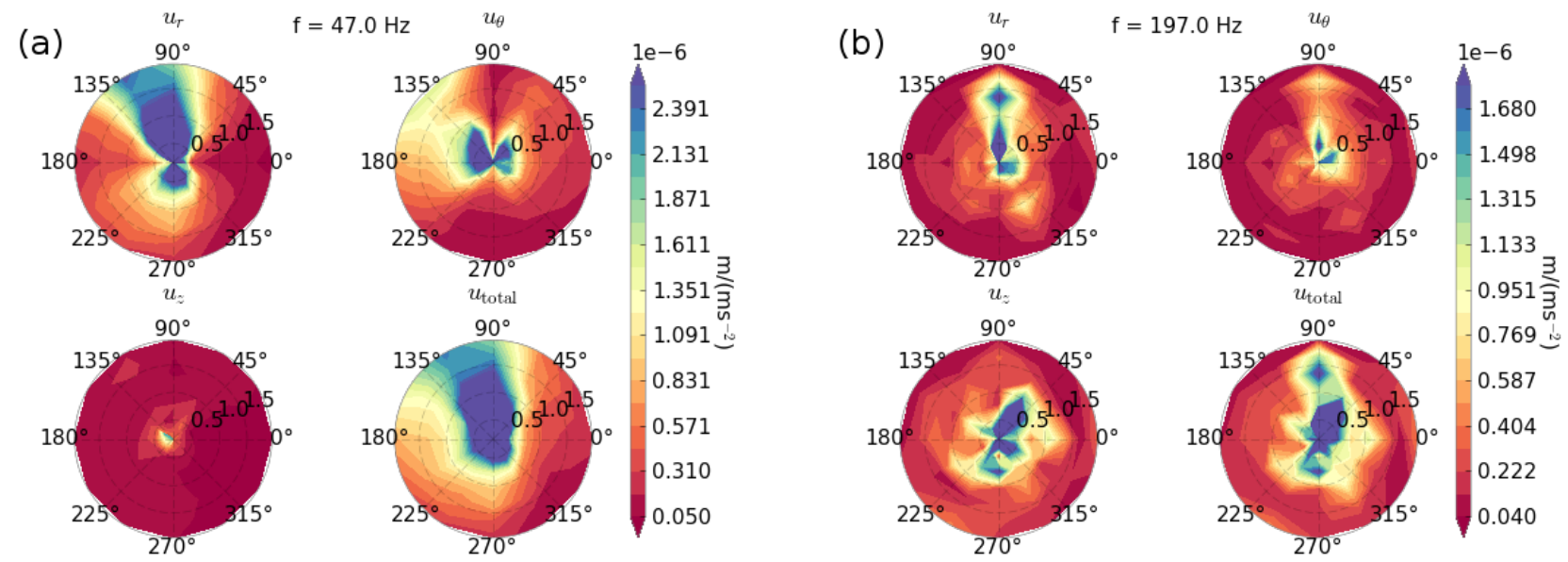

Figure 5. Illustrative magnitude maps for the circular array with axial excitation; the root is located along the 90 deg direction: (a) at $47 \mathrm{~Hz}$; (b) at $197 \mathrm{~Hz}$.
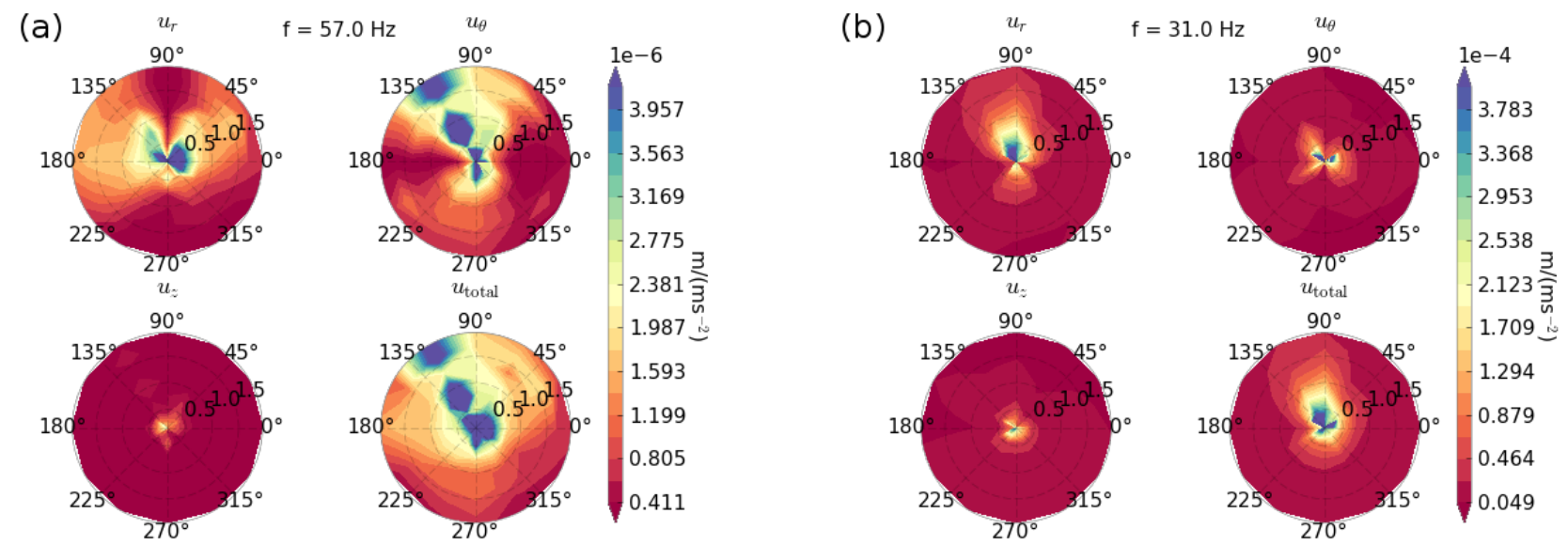

Figure 6. Illustrative magnitude maps for the circular array with transverse and vertical excitation; the root is located along the 90 deg direction: (a) transverse, at $57 \mathrm{~Hz}$; (b) vertical at $31 \mathrm{~Hz}$. 
quency response to vertical forcing indicates the location of the root correctly for both $u_{\text {total }}$ and $u_{r}$.

\section{Discussion and conclusions}

Illustrative results presented above prove vibration-based root mapping feasible. From all presented figures we clearly observe implications of the challenge which is inherent to the method as such - ground surface signals are a superposition of waves excited directly at the point where the excitation source is attached and waves re-radiated from the buried structures. Separating the two is often necessary to reveal the radiated component and it is not a straightforward task, given that the soil is rarely homogeneous. It is complicated even further by strong acoustic coupling between the soil and the root (owing to the material properties).

Whilst it is expected that advanced signal processing on the array readings would greatly enhance the performance of this method, analysing 'raw' ground surface responses as maps is to some extent attractive for its intuitiveness and simplicity. We note, that in this contribution only preliminary results are reported. Several important issues remain to be addressed in our future publications include application of array signal processing techniques and eliminating the effect of waves directly excited in the ground. Following from those, the method would need to be tested for more realistic configurations including more root branches.

Nevertheless, our contribution shows that tree roots leave a distinct signature on ground surface vibration responses, when excited at the trunk. Moreover, their location can be inferred preliminarily without advanced signal processing. Our hope is that after further developments, the method will be implemented in arboreal practice.

\section{Acknowledgement}

The support provided by the EPSRC (under grant $\mathrm{EP} / \mathrm{K} 021699 / 1$ : 'Assessing the Underworld') is gratefully acknowledged.

\section{References}

[1] L. Guo, J. Chen, X. Cui, B. Fan, H. Lin, Plant and Soil 362, 1 (2013)

[2] M. Amato, B. Basso, G. Celano, G. Bitella, G. Morelli, R. Rossi, Tree Physiology 28, 1441 (2008)

[3] R.W. Mankin, S.N. Johnson, D.V. Grinev, P.J. Gregory, Root Feeders: An Ecosystem Perspective. CABI Publishing, Wallingford, UK pp. 20-32 (2008)

[4] Y. Hirano, M. Dannoura, K. Aono, T. Igarashi, M. Ishii, K. Yamase, N. Makita, Y. Kanazawa, Plant and Soil 319, 15 (2009)

[5] L.F. Ow, E.K. Sim, Plant Biosystems - An International Journal Dealing with all Aspects of Plant Biology 146, 288 (2012)

[6] C.V.M. Barton, K.D. Montagu, Tree Physiology 24, 1323 (2004)

[7] J.M. Muggleton, M.J. Brennan, Y. Gao, Journal of Applied Geophysics 75, 54 (2011)

[8] J.M. Muggleton, E. Rustighi, Géotechnique Letters 3, 137 (2013)

[9] B.F. Wilson, The Growing Tree (Univ of Massachusetts Press, 1984), ISBN 0-87023-424-2

[10] M.K. Kalkowski, J.M. Muggleton, E. Rustighi, Journal of Sound and Vibration 390, 67 (2017) 